Beata Śmigielska

Université de Silésie, Katowice

Pologne

(D) https://orcid.org/0000-0002-3383-0030

\section{Modèles sémantico-syntaxiques des prédicats dans la conception de la grammaire à base sémantique de Stanisław Karolak quelques problèmes et solutions}

Semantic-syntactic models of predicates

in Stanisław Karolak's conception of semantic-based grammar some problems and solutions

\begin{abstract}
The author examines semantic-syntactic models of predicates according to Stanislaw Karolak's conception of semantic-based grammar. From the list of first-order bivalent predicates provided by S. Karolak, the author analyses some of them from the point of view of the number and quality of the implied argument positions. During the analysis it often turns out that more than one model refers to one form of predicate (one signifiant). This phenomenon is due to the polysemous nature of words in natural language and, therefore, to the ambiguity of uses of predicates in different contexts (more than one signifié). In order to determine the number and type of arguments semantically implied by a given predicate, the author applies the test of negation, contraposition, and paraphrase leading to a semantic decomposition and emphasises the role of the metaphor and metonymy in their interpretation.
\end{abstract}

\title{
Keywords
}

Predicate-arguments structure, semantic grammar, valence, paraphrase, decomposition, metaphor, metonymy 


\section{Introduction}

Stanisław Karolak, analysant des rapports entre le niveau sémantique et celui de la syntaxe, a présenté sa vision du fonctionnement de la langue connue sous le nom de grammaire à base sémantique et l'a spécifiée à travers la description de deux systèmes linguistiques : celui du polonais (cf. S. Karolak, 1984, 2001, 2002) et celui du français (cf. p. ex. : K. Bogacki, H. Lewicka, 1983 ; K. Bogacki, S. Karolak, 1991, 1992 ; S. Karolak, 1998, 2007 ; W. Banyś, 2019). Dans ses travaux, il essayait, et il l'a fait avec un grand succès, de faire la description de ces deux langues en y appliquant le calcul des prédicats et des propositions emprunté à la logique. Ainsi, les structures prédicats-arguments (SPA), qui se situent au niveau conceptuel, inaccessibles de manière directe aux locuteurs des langues, sauf si l'on soumet leurs réalisations à des tests linguistiques, constituent le fondement de sa conception de grammaire. La construction des modèles des structures prédicats-arguments est la question la plus compliquée dans la grammaire à base sémantique, ce que souligne aussi l'auteur de l'approche. Pour résoudre ce problème, il propose quelques solutions parmi lesquelles la décomposition sémantique en éléments plus simples est celle à laquelle il donne la primauté (S. Karolak 2002, 2007 ; cf. aussi p. ex. B. Śmigielska 2013, 2019). Même s'il se rend compte du fait que cette opération est subjective, il la croit la plus efficace. Cette procédure suscite depuis toujours l'intérêt des linguistes qui travaillent sur le problème de la valence des prédicats, et elle a aussi bien des partisans (cf. p. ex. A. Bogusławski, 1974, 1981 ; M. Danielewiczowa, 2010, 2017) que des adversaires (cf. p. ex. M. Grochowski, 1984 ; A. Przepiórkowski, 2017). Les premiers mettent en évidence une grande efficacité de la décomposition sémantique, malgré sa complexité, tandis que les autres soulignent son caractère trop intuitif, donc peu sûr.

Dans ce qui suit, dans la première partie du texte, nous voudrions rappeler très brièvement les notions les plus importantes de l'approche mentionnée, pour passer ensuite à l'analyse de quelques prédicats choisis, particulièrement de ceux qui posent des problèmes de classement suivant les critères de valence et d'ordre.

\section{Notions fondamentales de la grammaire à base sémantique}

Les prédicats représentent une sorte de « situations », de « relations », reflétant tout ce qui se passe dans la réalité non linguistique et ils ouvrent un certain nombre de positions pour des arguments. Ces derniers peuvent être appelés «participants » qui prennent part à ces situations, à ces relations-là. Pour présenter la 
structure prédicat-argument(s), p. ex. de se marier et de proposer, il faut, en premier lieu, déterminer combien de positions d'arguments ces prédicats impliquent et de quels types ils sont. Rappelons qu'il y en a deux types : arguments d'objets, qui réfèrent à un objet ou à un ensemble d'objets de la réalité extralinguistique, et arguments propositionnels, qui réfèrent à des situations, qu'elles soient des états, des événements, des actions ou des processus. Les prédicats, selon le classement qualitatif (suivant le type d'arguments impliqués), sont donc de premier ordre ou d'ordre supérieur (de deuxième ordre). Les premiers n'ouvrent leurs positions que pour les arguments d'objets ( $\mathrm{x}, \mathrm{y}, \mathrm{z}, \mathrm{v})$, et les deuxièmes impliquent au moins un argument propositionnel ( $\mathrm{p}, \mathrm{q}, \mathrm{r}, \mathrm{s})$. Il est donc crucial de déterminer le nombre des positions d'arguments que les prédicats ouvrent. Ainsi, selon le classement quantitatif, les prédicats peuvent ouvrir une position d'argument (monovalents), deux positions d'arguments (bivalents), trois positions d'arguments (trivalents) et quatre positions d'arguments (tétravalents) (S. Karolak 1984, 2007 ; cf. aussi p. ex. A. Czekaj, B. Śmigielska, 2009 ; I. Pozierak-Trybisz, 2009).

Revenons donc aux modèles sémantico-syntaxiques des prédicats. Les symboles logiques que nous allons utiliser sont ceux dont S. Karolak se servait dans la dernière version de la grammaire à base sémantique de 2007 (cf. p. ex. : M. Hrabia, 2011 ; B. Śmigielska, 2013).

1. se marier - ce prédicat implique deux positions d'arguments, il est bivalent $\mathrm{du} \mathrm{I}^{\mathrm{er}}$ ordre, car la première et la deuxième position sont réservées pour des arguments d'objets : qqn se marie avec qqn. Le modèle sémantico-syntaxique serait donc le suivant :

$$
\mathbf{P}(\mathbf{x}, \mathbf{y})
$$

et il peut être représenté par la phrase suivante :

\section{Anne s'est mariée avec Pierre.}

Les deux positions d'argument ouvertes par le prédicat en question sont remplies à la surface par deux indices (Anne et Pierre).

2. proposer - ce prédicat ouvre trois positions d'argument : qqn propose qqch. $\grave{a} q q n$, mais cette fois-ci deux pour des arguments d'objets et une pour un argument propositionnel. Le prédicat proposer est donc trivalent du $\mathrm{II}^{\mathrm{e}}$ ordre. Le modèle sémantico-syntaxique serait donc comme suit :

$$
\mathbf{P}(\mathbf{x}, \mathbf{q}, \mathbf{z})
$$

et il peut être représenté par la phrase du type p. ex. : 
Je propose à Marie de passer cette soirée avec moi,

où la première position et la troisième (les appellations : première, deuxième, etc. sont tout à fait conventionnelles) sont remplies par les indices (Je, Marie) et la deuxième, étant ouverte pour un argument propositionnel, renvoie à une situation (passer cette soirée avec moi), c'est-à-dire à une nouvelle structure prédicat-argument(s) où le prédicat passer crée sa propre structure prédicatargument(s).

\section{Problèmes de classement des prédicats}

S. Karolak a établi les listes des exemples des prédicats selon les critères de valence et d'ordre mentionnés ci-dessus. Analysons donc, de ce point de vue, le classement de certains prédicats :

La liste des prédicats bivalents du $\mathrm{I}^{\mathrm{er}}$ ordre (S. Karolak, 2007 : 117—118) :

abandonner, accompagner, agresser, aimer, allumer, améliorer, apercevoir, arranger, arrêter, attirer, avoir, briser, brosser, brûler, boire, casser, collectionner, conduire, construire, contenir, coucher, couvrir, courir, cuir, découvrir, décrocher, détester, détruire, diffuser, dominer, écrire, élaborer, embaucher, empoisonner, emprisonner, enfoncer, enregistrer, épouser, établir, éteindre, fabriquer, fermer, former, fricasser, frotter, gagner, garder, habiller, habiter, habituer, haï, illuminer, inventer, laver, licencier, manger, nettoyer, occuper, ouvrir, pêcher, pendre, perdre, piller, prendre, préparer, quitter, raccrocher, ranger, rappeler, raser, regarder, représenter, réveiller, rosser, saccager, suivre, supporter, toucher, tourner, tromper, vider, viser, visiter.

Si l'on regarde les prédicats listés ci-dessus, ils semblent être tous bivalents du $\mathrm{I}^{\mathrm{er}}$ ordre. Soumettons donc quelques-uns d'entre eux à une analyse plus détaillée. En proposant les modèles sémantico-syntaxiques des prédicats, nous allons baser sur des exemples de phrases trouvés sur Internet.

\subsection{Perdre - gubić/zgubić, p. ex. :}

a) Il y a 17 ans, un Écossais a perdu son portefeuille après une soirée dans un bar dans la ville de Dunfermline.

b) Monsieur Picot a perdu ses clefs au zoo.

c) Il jure avoir perdu sa femme au stade et espère la retrouver. 
Puisque deux positions ouvertes par le prédicat perdre sont des arguments d'objets (remplies ici par : portefeuille, clefs, femme), le prédicat est bivalent du ${ }^{\text {er }}$ ordre. Ainsi, le modèle sémantico-syntaxique de perdre serait le suivant :

$\mathbf{P}(\mathbf{x}, \mathbf{y})$ - prédicat bivalent du $\mathrm{I}^{\mathrm{er}}$ ordre où « $x$ » et « $y$ » sont des variables à remplir par des objets, c.-à-d., ils réfèrent aux objets.

Analysons ci-dessous la même forme superficielle prédicative employée dans un autre contexte pour vérifier si son modèle logique serait le même que le précédent.

\subsection{Perdre - tracić/stracić, p. ex. :}

a) Antoine Leiris a perdu sa femme, Hélène Muyal-Leiris, le 13 novembre 2015, assassinée au Bataclan.

b) Le chien qui perd la vue s'habitue peu à peu à son sort.

c) Arrivé au Tonkin à une époque de persécution violente, il a vécu pendant des mois dans des cachettes, sans perdre sa bonne humeur.

d) Ronaldo n'a pas perdu sa patience.

À base des exemples ci-dessus, on observe deux régularités suivantes : le prédicat perdre implique sémantiquement une position d'argument d'objet (ici, p. ex. : chien, arbre, général, Ronaldo, etc.), une position d'argument propositionnel. Rappelons que, selon la définition karolakienne, si une position d'argument d'objet est saturée par une expression référentielle (indices : noms propres, pronoms personnels ; descriptions définies), elle réfère de manière directe et univoque à un objet concret ou à un ensemble d'objets concrets de la réalité extralinguistique (S. Karolak, $2007: 25$ ). Ainsi, il est évident que toutes les notions abstraites qui remplissent la deuxième position d'argument (ici, p. ex. : vue, patience, bonne, humeur), sont des arguments propositionnels du prédicat analysé, donc le prédicat perdre serait dans ce cas-ci bivalent du $\mathrm{II}^{\mathrm{e}}$ ordre :

$\mathbf{P}(\mathbf{x}, \mathbf{q})$ - prédicat bivalent du $\mathrm{II}^{\mathrm{e}}$ ordre où « $x$ » est une variable représentant un argument d'objet, tandis que « $q »$ représente une position ouverte pour un argument propositionnel.

Il est intéressant de comparer deux phrases :

1. Il jure avoir perdu sa femme au stade et espère la retrouver. (gubić/zgubić)

- Antoine Leiris a perdu sa femme, Hélène Muyal-Leiris, le 13 novembre 2015, assassinée au Bataclan. (tracić/stracić) 
Dans les phrases ci-dessus, en deuxième position apparaît le mot femme qui, par défaut, typiquement, peut être traité comme un concret. Il est vrai que, si la même expression linguistique possède deux sens différents, il y a une différence entre eux à dégager. Lorsque l'on voit ces deux phrases, le dicton bien connu nous vient à l'esprit, notamment : « il ne faut pas se fier aux apparences ». Étant donné que la langue peut nous tromper souvent en cachant le vrai sens d'une phrase derrière une structure superficielle, il est bien d'essayer de trouver un moyen de remédier à ce problème et d'y faire face.

Afin d'arriver au sens de l'expression perdre une femme, il faudrait l'interpréter dans le contexte (cf. p. ex. J. Firth, 1957 ; I. Mel'čuk, A. Žolkovskij, 1970 ; I. Mel'čuk et al., 1981 ; J.-P. Declés, 1997 ; A. Kilgariff, 1997 ; G. Gross, 1999a ; W. Banyś, 2005) et en faire une paraphrase. Dans l'exemple « 1 » le prédicat perdre (gubić/zgubić) veut dire plus ou moins que : $x$ a/possède $y$ et puis $x$ n'al possède plus $y$ et $x$ n'a pas $y$, donc les deux positions « $x$ » et « $y$ » sont des positions arguments d'objet. En plus, le contexte de la phrase indique que la situation de perte de la femme se fait au stade et que $\langle x$ » ne peut pas retrouver $\langle y$ ». L'exemple « 2 » est beaucoup plus compliqué à interpréter, car le mot femme qui fait penser à un objet en fait ne l'y renvoie pas directement. Perdre une femme (stracić żone) représente une expression de type métaphorique (du point de vue de l'expression concrète apparaissant à la surface on pourrait dire de type métonymique) et c'est une situation très fréquente que l'on parle par métaphores dans notre communication (cf. p. ex. G. Lakoff, M. Johnson, 2003), ce qui influence de manière significative l'analyse du type prédicat-argument(s). Alors, pour que l'on puisse proposer le modèle sémantico-syntaxique des constructions analysées, avec des expressions du type métaphorique, il est nécessaire de les paraphraser. Il suffit donc de proposer une autre phrase sémantiquement équivalente à celle de départ. P. ex. :

2. Antoine Leiris a perdu sa femme, Hélène Muyal-Leiris, le 13 novembre 2015, assassinée au Bataclan. (tracić/stracić) — phrase de départ

- Hélène Muyal-Leiris, la femme d'Antoine Leiris est morte le 13 novembre 2015, assassinée au Bataclan. - exemple de paraphrase

Il est à souligner que l'opération du paraphrasage permet de comprendre ce qui est réellement dit dans la phrase. Grâce à la paraphrase, il est possible de constater que, dans l'exemple analysé, la deuxième position d'argument est ouverte non pas pour un argument d'objet, même si le mot femme paraît représenter un concret, mais elle implique une position d'argument propositionnel qui renvoie ici à l'événement de la mort de la femme dont le mot femme n'est qu'un élément évocateur renvoyant à une partie d'un tout. Alors, le modèle sémantico-syntaxique de l'expression perdre sa femme (stracić żonę) serait le même que dans le cas général du prédicat perdre + abstrait : $\mathbf{P}(\mathbf{x}, \mathbf{q})$. 


\subsection{Perdre - przegrać/przegrywać, p. ex. :}

a) Ses deux défaites comprises, il n'a perdu que 27 sets en 100 matches.

b) Il avait perdu en finale en 2013 contre Novak Djokovic.

c) Monaco : Il perd 3 millions d'euros au casino en une heure.

Pour décider de la valence et de l'ordre du prédicat perdre au sens employé dans les phrases plus haut, il est bien de faire recours au contexte où il peut être utilisé. Pour perdre quelque chose, quoi que ce soit, dans les contextes ci-dessus, il faut participer à une sorte de jeu ou de compétition (ici : matches, finale, (jeu au) casino). Si l'on y participe, on a un ou des rivaux à battre dans le but d'obtenir un avantage ou un bien. Alors, la décomposition sémantique de ce prédicat serait p. ex. : $x$ joue à qqch. avec $y$; on gagne ce pour quoi ils jouent si l'on atteint un résultat exigé ou meilleur que les autres, mais x n'atteint pas le résultat exigé ou meilleur que les autres, donc $x$ ne reçoit pas $q$. Étant donné l'analyse du contexte et du sens du prédicat en question, on arrive à sa forme logique suivante :

$\mathbf{P}(\mathbf{x}, \mathbf{q}, \mathbf{y})$ - prédicat trivalent du $\mathrm{II}^{\mathrm{e}}$ ordre où $« x »$ et $\langle y »$ sont des positions d'arguments d'objets et « $q$ » est une position d'argument propositionnel.

Après avoir fait les analyses du prédicat perdre, on voit que les prédicats ayant la même forme superficielle peuvent avoir des modèles sémantico-syntaxiques complètement différents : $1-\mathrm{P}(\mathrm{x}, \mathrm{y})-$ gubić/zgubić, $2-\mathrm{P}(\mathrm{x}, \mathrm{q})-$ tracić/ stracić, $3-\mathrm{P}(\mathrm{x}, \mathrm{q}, \mathrm{y})$ - przegrać/przegrywać (les traductions en polonais ou une autre langue le confirment d'une manière supplémentaire, la confrontation des emplois d'un mot avec ses traductions dans d'autres langues est d'ailleurs l'un des éléments clés de l'Approche Orientée Objets dans la discussion sur le nombre de sens d'un mot dans une langue donnée et leur désambiguïsation (cf. p. ex. W. Banyś, 2002a, 2002b, 2005)). Naturellement, ce phénomène résulte de la polysémie de la langue, qui caractérise généralement la plupart des unités linguistiques des langues naturelles et en particulier celles qui sont les plus fréquentes. Alors, avant de classifier les prédicats selon le nombre des positions d'arguments qu'ils ouvrent et leur type, il ne faut pas oublier de prendre en considération leur valeur polysémique possible.

Soumettons maintenant à l'analyse deux prédicats suivants de la liste karolakienne : aimer et détester. Sont-ils bivalents du $\mathrm{I}^{\mathrm{er}}$ ordre ? Voici quelques phrases représentatives, p. ex. : 


\subsection{Aimer - lubić/kochać \\ Détester — nienawidzić, p. ex. :}

a) Marie aime/déteste les jupes.

b) Mes parents aiment/détestent les montagnes.

c) Il aime/déteste le chocolat.

d) Leurs amis aiment/détestent le vélo.

e) Jacques aime/déteste cette femme.

Les prédicats aimer et détester sont sûrement bivalents, donc ils ouvrent deux positions d'arguments, mais la question qui se pose est de savoir quels sont leurs types. Il est évident que la première position d'argument est une position d'argument d'objet, mais pour déterminer la qualité de la deuxième position d'argument, il faudrait faire les paraphrases des exemples cités ci-dessus. P. ex. :

a) Marie aime/déteste les jupes.

- Marie aime/déteste porter/nettoyer/repasser, etc. les jupes.

b) Mes parents aiment/détestent les montagnes.

- Mes parents aiment/détestent grimper sur les/regarder les/passer le temps aux, etc. montagnes.

c) Il aime/déteste le chocolat.

- Il aime/déteste manger/déguster/acheter, etc. du chocolat.

d) Leurs amis aiment/détestent le vélo.

- Leurs amis aiment/détestent réparer le/nettoyer le/faire du/vélo.

e) Jacques aime/déteste cette femme.

- Jacques aime/déteste regarder/penser à/être en compagnie de, etc. cette femme.

Le test de paraphrase prouve que la deuxième position d'argument, même si elle est remplie à la surface par des noms renvoyant typiquement aux objets concrets (jupes, montagnes, chocolat, vélo, femme), n'est pas une position d'argument d'objet, mais elle est une position d'argument propositionnel. Il s'avère que l'argument propositionnel réfère à une nouvelle structure prédicat-argument(s), qui se cache à la surface sous une expression renvoyant typiquement à un objet, qui remplit, à son tour, l'une des positions d'argument de la structure prédicatargument(s) impliquée. Cette expression est une sorte de métonymie - synecdoque - renvoyant à une partie d'un tout au lieu de renvoyer directement au tout.

La question de savoir pour quelle raison nous parlons comme cela, est une autre question, très intéressante, débattue dans entre autres les traités sur la rhétorique (cf. p. ex. P. Fontanier, 1977 [1821-1827] ; H. Morier, 1998)

Dans de tels cas, comme dans tous les cas d'emploi de type métaphorique ou métonymique pareils, la langue peut nous induire en erreur quant à la réelle struc- 
ture sémantique de la phrase et c'est à l'aide des paraphrases que l'on peut arriver à ce qui y est réellement dit (cf. B. Śmigielska, 2013, 2019 ; A. Czekaj, 2018). Étudier minutieusement la valence des prédicats dans le cadre de la grammaire à base sémantique de ce point de vue est une tâche à faire, ce qui aura un effet non seulement sur les qualifications particulières des prédicats du point de vue de la valence, mais aussi, p. ex. sur la manière de concevoir (le continuum de) la dimension des verbes support (cf. p. ex. G. Gross, 1993, 2012 ; R. Vivès, 1993 ; G. Vetulani, 2000, 2012 ; A. Czekaj, 2013).

Ainsi, le modèle sémantique des prédicats aimer et détester serait comme suit :

$\mathbf{P}(\mathbf{x}, \mathbf{q})$ - prédicat bivalent du $\mathrm{II}^{\mathrm{e}}$ ordre où la première position d'argument est une position d'argument d'objet et la deuxième position d'argument est une position d'argument propositionnel.

Le prédicat suivant qui se trouve sur la liste des bivalents du $\mathrm{I}^{\mathrm{er}}$ ordre, et, qui paraît intéressant à étudier, est le prédicat écrire (cf. p. ex. A. Bogusławski, 2017). Regardons ses emplois dans les phrases suivantes:

\section{5. Écrire — pisać/napisać, p. ex. :}

a) Elle écrit un livre et réalise un rêve d'enfant.

b) J'ai écrit une trilogie de Fantasy.

c) Je vais écrire un roman pour enfants. / Je vais écrire un roman sur les enfants pour enfants/pour adultes.

d) Il écrit une comédie pour le théâtre intitulé "Les Pantins".

e) Avez-vous écrit la lettre au Père Noël ?

f) As-tu écrit une carte postale à tes amis?

Dans les phrases ci-dessus, la première position d'argument est ouverte pour un argument d'objet. Quant à la deuxième position, la situation est beaucoup plus complexe. À la surface, les expressions qui remplissent cette position d'argument sont de type, p. ex. : livre, lettre, trilogie, roman, carte postale, comédie, donc, au premier coup d'œil, typiquement, elles renvoient aux objets plutôt concrets (comédie y a un statut plutôt ambigu). Cependant, on a affaire aux expressions qui sont polysémiques (cf. p. ex. B. Śmigielska, 2011). Expliquons-le à l'exemple du mot livre. Livre - ksiażka (et toutes les autres expressions énumérées plus haut) a deux sens différents.

Le premier livre - książka réfère à l'objet physique que l'on peut p. ex. déchirer, brûler, jeter dans la poubelle ou par la fenêtre, etc. Le deuxième livre - książa, à son tour, renvoie au contenu que l'on peut lire, écrire, comprendre, 
traduire, etc. (il est bien visible que d'autres prédicats, associés au livre, aident à distinguer ces deux sens).

Le problème est que les deux sens de livre ont le même signifiant. Il en est de même avec d'autres expressions de ce type et cela peut être, sans aucun doute, trompeur. Alors, pour décider si la deuxième position de prédicat écrire renvoie à un objet ou à un contenu, il serait bien d'analyser les expressions qui la remplissent du point de vue de leur référence et leur sens (Ch. K. Ogden, I. A. Richards, 1923 ; cf. aussi K. Heger, 1969) et d'en faire les paraphrases. P. ex. :

a) Elle écrit un livre et réalise un rêve d'enfant.

- Elle écrit un texte d'une certaine longueur sur un assemblage de feuilles qui est appelé livre.

- Elle a écrit des signes ayant certains sens (un texte) sur un assemblage de feuilles de papier qui est appelé livre.

b) J'ai écrit une trilogie de Fantasy.

- J'ai écrit un texte d'une certaine longueur sur un assemblage de feuilles qui est appelé trilogie de Fantasy.

c) Je vais écrire un roman pour enfants.

- Je vais écrire un texte d'une certaine longueur sur un assemblage de feuilles qui est appelé roman.

d) Il écrit une comédie pour le théâtre intitulé "Les Pantins".

- Il écrit un texte d'une certaine longueur sur un assemblage de feuilles qui est appelé comédie.

e) Avez-vous écrit la lettre au Père Noël ?

- Avez-vous écrit un texte d'une certaine longueur sur une (des) feuille(s) qui est appelée lettre.

f) As-tu écrit une carte postale à tes amis?

- As-tu écrit un texte d'une certaine longueur sur une carte portant généralement une photo ou une illustration, qui est appelé carte postale.

Dans le cas des phrases ci-dessus $(a-f)$, on n'a pas affaire aux objets, mais au contenu et, en conséquence, la deuxième position du prédicat écrire est un argument propositionnel.

Le problème suivant à résoudre est lié à la valence du prédicat écrire. Est-il bivalent ou trivalent ? Dans les phrases $\langle c, d, e, f »$, on voit les expressions, précédées de prépositions «pour 》 ou " à », compléter le sens du prédicat en question, telles que, p. ex. : pour femmes, pour le théâtre, au Père Noel, à tes amis.

Il est intéressant de comparer de ce point de vue les constructions en « $\grave{a} »$ et en «pour » aussi bien en français qu'en polonais :

Il a écrit un livre/une comédie pour enfants? / Il a écrit un livre/une comédie aux (à ses) enfants. 
Napisal książkę/komedię dla dzieci? / Napisat książkę/komedię (swoim) dzieciom? / Napisal ksiązkę/komedię dla dzieci (swoim) dzieciom.

$v S$

? Il a écrit une lettre/une carte postale pour enfants / Il a écrit une lettre/une carte postale aux (à ses) enfants.

? Napisal list/pocztówkę dla dzieci / Napisat list/pocztówkę (swoim) dzieciom/ do (swoich) dzieci.

Étant donné que quelqu'un écrit un certain contenu et le présente sous forme de livre, roman, comédie, trilogie ou lettre, carte postale, il ne le fait d'habitude ni pour lui-même ni à lui-même (même si ce n'est pas naturellement exclu). D'où les phrases absolument naturelles du type, p. ex. : Il a écrit une lettre aux enfants. Le caractère moins naturel, mais non pas exclu, de Il a écrit un livre aux enfants consiste en ceci qu'un livre diffère d'une lettre par sa longueur et typiquement concerne un public non spécialement déterminé, et par conséquent, typiquement, on n'explicite pas ceux auxquels le livre est adressé, généralement c'est le grand public, mais on peut naturellement envisager la situation où le père a l'intention d'écrire une lettre à ses enfants, mais il l'écrit au fur et à mesure si longue qu'il leur a écrit en fin de compte tout un livre, et dans cette situation les phrases du type Il a écrit un livre aux enfants seraient parfaitement acceptables.

Dans le cas d'une lettre donc, à la différence d'un livre, normalement, le public est déterminé, mais on peut naturellement avoir aussi un public général, comme c'est le cas p. ex. des lettres qui sont ouvertes.

Analysons encore les phrases ci-dessous :

Il a écrit un livre pour enfants.

Il a écrit un livre pour enfants à ses enfants sur les dangers dans l'eau.

Napisal swoim dzieciom ksiażkę dla dzieci o niebezpieczeństwach czyhajacych $w$ wodzie.

$v S$

Il a écrit une lettre pour enfants à ses enfants sur les dangers dans l'eau.

Napisat list dla dzieci do swoich dzieci/swoim dzieciom o niebezpieczeństwach czyhajacych $w$ wodzie.

Si l'on peut peut-être sentir une certaine maladresse de certaines phrases cidessus, elle n'est pas le résultat d'une différence dans leur structure sémantique, mais dans les associations typiques que l'on partage généralement quant aux caractéristiques des lettres, livres, cartes postales, etc., une influence plutôt « pragmatique » que sémantique.

Quant à la différence donc entre "pour » et « $\grave{a} »$, elle consiste en ceci que "pour» dans p. ex. pour enfants exprime la caractéristique générale du contenu du livre, de la comédie, de la carte postale, etc. (à la différence de «sur» dans 
p. ex. Il a écrit un livre pour enfants sur les dangers dans l'eau qui spécifie davantage cette caractéristique générale du livre) et « $a ̀ »$ exprime les personnes auxquelles le livre, la comédie, la carte postale ont été envoyés ou offerts.

C'est que, en dernière instance, écrire ce n'est rien d'autre que dire/parler, c'est seulement le matériel (matière sonore ou graphique) et la longueur (parole, discours ne sont pas normalement trop longs, par contre ce que l'on écrit peut être aussi bien long que court) qui changent. Dans le cas de dire/parler et écrire, on a donc la même structure sémantique profonde : qqn dit qqch. à qqn à propos de qqch. ou de qqn et les réalisations superficielles à l'aide de dire/parler/écrire, etc. mettent en relief à la surface différents éléments de cette structure sans qu'il soit impossible de les développer davantage pour qu'elles réalisent toutes leurs positions d'argument.

Remarquons que ce type d'analyse est possible si l'on choisit, parmi les trois conceptions générales des analyses en structures prédicat-argument(s) (type A. Bogusławski et sa vision de la structure prédicat-arguments : "plus de trois ou trois au plus » (1981:3) concernant le nombre des arguments impliqués par les prédicats ; type G. Gross et la fameuse définition du prédicat comme « la suite la plus longue de ses arguments » (1999b : 113); et type S. Karolak, p. ex. 1984, 2007 - intermédiaire entre les deux), la position intermédiaire mixte : A. Bogusławski - S. Karolak, fondée principalement sur une application cohérente et conséquente des paraphrases et des contradictions.

Cf. donc p. ex. parler vs dire vs écrire :

Il a dit à Marc que le conflit est imminent (Il a dit à Marc à propos du conflit qu'il est imminent).

$v S$

Il a parlé avec Marc (à propos) de l'imminence du conflit.

Il a parlé avec Marc (à propos) du conflit disant qu'il est imminent.

$v S$

Il a écrit un livre sur l'imminence du conflit.

Il a écrit un livre sur le conflit disant qu'il est imminent.

Il a écrit dans sa lettre aux enfants à propos du conflit qu'il est imminent.

Il a écrit dans sa lettre aux enfants à propos de Marc qu'il est ridicule.

Il a écrit dans sa lettre aux enfants à propos du comportement de Marc qu'il est ridicule.

$v S$

Napisal w swoim liście do dzieci o konflikcie, że zaraz wybuchnie.

Napisal w swoim liście do dzieci o Marku, że to co robi, jest śmieszne.

Napisal w swoim liście do dzieci o zachowaniu Marka, że jest śmieszne.

La décomposition des prédicats en question aurait donc la forme, à l'aide de dire, dire étant un des primitifs sémantiques, suivante : Parler $=x$ dit à $y$ 
à propos de $r$ que $s$. Dans le cas de parler, « $r$ » est exprimé typiquement à la surface par un seul syntagme nominal (du conflit, de la situation, etc.), qui peut spécifier aussi « $s$ » en même temps (de l'imminence du conflit, de la terrible situation, etc.). Dans le cas d'écrire, « $r$ » est spécifié typiquement par un syntagme nominal cumulant en lui aussi bien le contenu non spécifié (qui peut être spécifié par l'ajout de p. ex. de, sur, à propos de, etc.) que la forme (lettre, livre, etc.), « $s$ » étant spécifié éventuellement par une caractéristique ajoutée au syntagme (du type : sur l'imminence du conflit, etc.) ou par une proposition subordonnée (disant que...). Et, dans le cas de dire, « $r$ » et « $s »$ sont typiquement exprimés par des syntagmes nominaux (p. ex. je te dis la vérité sur la situation) ou syntagme nominal et une proposition subordonnée ou une proposition subordonnée seule.

Ce sont là des différences superficielles de la réalisation de la même structure sémantique du point de vue adopté, position mixte A. Bogusławski - S. Karolak, pour les analyses des structures prédicat-arguments (cf. aussi avec la position d’A. Bogusławski (2017 : 39) : " Parmi les marquages des types de prises de parole de base, les marquages fondus dans la langue durkheimienne-saussurienne elle-même, les marquages distincts des concepts terminologiques individuels, les descriptions basées sur le verbe polonais 'powiedział' ['a dit'] et ses équivalents de traduction, tels que le mot anglais 'said', se distinguent de manière assez évidente » (traduit par l'auteure du texte)).

Ajoutons que les prédicats écrire, parler et dire font partie du grand champ des prédicats de communication qui sont à analyser selon la structure profonde du prédicat sémantiquement primitif qu'est dire (cf. p. ex. A. Wierzbicka, $2006: 185$ ) et leur structure superficielle différenciée a été très bien étudiée dans les travaux dans le cadre du Lexique-Grammaire (cf. p. ex. J. Giry-Schneider, 1994 ; R. Vivès, 1998 ; I. Eshkol, D. Le Pesant, 2007). Il serait bien de joindre les deux types d'analyses dans une description complète des passages et de leurs contraintes de la structure profonde des prédicats de communication proposée à leur réalisation superficielle si bien documentée dans le cadre des travaux du Lexique-Grammaire.

Parler et écrire, à la différence de dire, permettent de ne pas dire ce que l'on pense à propos de quelque chose et de nous limiter à dire seulement de quoi on parle ou écrit, ce qui n'est pas possible dans le cas des structures superficielles de dire, cf. p .ex. :

$?^{X}$ Il a dit à Marc que le conflit.

Il a parlé du conflit.

Il a écrit un livre sur le conflit.

Analysons encore les phrases ci-dessous :

Il a écrit un livre pour enfants. 
Il a écrit un livre pour enfants à ses enfants sur les dangers dans l'eau.

Napisal swoim dzieciom książkę dla dzieci o niebezpieczeństwach czyhajacych $w$ wodzie.

$v S$

Il a écrit une lettre pour enfants à ses enfants sur les dangers dans l'eau.

Napisat list dla dzieci do swoich/swoim dzieciom o niebezpieczeństwach czyhajacych $w$ wodzie.

qui, comme on a souligné plus haut, pourraient faire sentir une certaine maladresse résultant de traits typiques qui caractérisent lettre, livre, carte postale, etc.

Autant dire que le modèle sémantico-syntaxique du prédicat analysé, dans l'optique adoptée, est comme suit :

$\mathbf{P}(\mathbf{x}, \mathbf{y}, \mathbf{r}, \mathbf{s})$ - prédicat tétravalent du $\mathrm{II}^{\mathrm{e}}$ ordre où la première et la deuxième position d'argument est ouverte pour un argument d'objet tandis que la troisième et la quatrième position d'arguments pour des arguments propositionnels.

Regardons encore quelques phrases avec le prédicat écrire :

\section{6. Écrire - pisać/napisać/zapisać, p. ex. :}

a) C'est écrire des mots d'amour sur des ballons.

b) Il a écrit les chiffres sur un chèque.

c) Les contemporains de Shakespeare ont écrit leur nom de famille de plusieurs façons différentes.

d) L'application pour écrire sur les photos Canva est disponible gratuitement sur l'Apple Store pour iPhone et sur Google Play pour Android.

e) Indiquez l'adresse et écrivez-la au dos de la carte, dans la zone à droite prévue à cet effet.

f) Croyez-moi ou non, Dieu me l'a écrit sur le mur en lettres rouges.

g) Et pour terminer, on a écrit sur l'ordinateur ce qu'on avait fait comme travail.

Prenons le cas des phrases ci-dessus du point de vue de la valence du prédicat écrire, qui semble représenter une structure prédicat-argument(s) différente par rapport à la précédente. Il est évident que la deuxième position d'argument, qui est remplie de type p. ex. : mots, chiffres, nom, adresse, est une position d'argument d'objet. Si l'on essaye d'associer d'autres prédicats à ces expressions-là, et en particulier à ceux qui mettent en évidence leur caractère d'objet, p. ex. effacer, barrer, 
souligner, gommer, etc., on voit qu'elles réfèrent aux objets de la réalité extralinguistique. En plus, dans presque toutes les phrases, sauf dans la phrase « 3.6. $c$ », apparait une information concernant le support sur lequel on écrit, p. ex. : sur des ballons, sur un chèque, sur les photos, au dos de la carte, sur le mur, sur l'ordinateur. La question est donc de savoir si c'est une position d'argument ouverte par le prédicat écrire et remplie par des expressions de lieu, ou, peut-être, ce sont des éléments adjoints, qui ne complètent que l'idée portée par le prédicat, mais qui ne sont pas nécessaires du point de vue du sens qu'il représente. Pour répondre à cette question, il faudrait analyser plus précisément ce que veut dire écrire dans le cas des contextes ci-dessus. Si l'on écrit, c'est-à-dire que l'on trace des signes et on le fait toujours sur un support. Et, en appliquant le test de négation logique à l'une de ces phrases, p. ex. :

b) Il a écrit les chiffres sur un chèque.

$v S$

*Il a écrit les chiffres sur rien.

*Il n'a pas écrit les chiffres sur quoi que ce soit.

il s'avère que la phrase niée est sémantiquement inacceptable, dans la lecture normale des sens des mots et si la phrase est non marquée du point de vue de l'intonation, puisque l'on ne peut pas écrire qqch. "sur rien », on l'écrit toujours sur quelque chose, sur un support matériel. Il en résulte que les expressions locatives de ce type - à la différence des expressions locatives du type dans la chambre, au restaurant, au parc, etc. dans les phrases du type p. ex. Il a écrit les chiffres sur un chèque dans la chambre, au restaurant, au parc — sont des éléments sémantiquement nécessaires, et par conséquent, ils remplissent l'une des positions d'arguments impliquées. C'est seulement dans la phrase «3.6. $c$ » que le support matériel d'écriture n'est pas précisé et cette position d'argument n'est pas saturée à la surface. Elle est facultativement saturable, c'est-à-dire qu'elle existe au niveau profond de la langue, et son apparition dans la phrase est toujours possible, mais non pas obligatoire. Cela dépend de l'intention du locuteur de transmettre telle ou telle information à son interlocuteur, d'autant plus que la phrase parle des contemporains de Sheakspeare, sous-entendu : tous/beaucoup/la majorité, etc., ce qui autorise ce type d'omission. On le voit directement si l'on prend la phrase «3.6. $c$ », où la position d'argument en question n'a pas été réalisée à la surface, et la remplir par une expression locative quelconque, p. ex. :

c) Les contemporains de Shakespeare ont écrit leur nom de famille de plusieurs façons différentes.

$v S$

- Les contemporains de Shakespeare ont écrit leur nom de famille de plusieurs façons différentes sur les documents officiels. 
Ainsi, le modèle logique du prédicat écrire employé dans les contextes ci-dessus (cf. $6 a-6 g$ ) est le suivant :

$\mathbf{P}(\mathbf{x}, \mathbf{y}, \mathbf{z})$ - prédicat trivalent du $\mathrm{I}^{\mathrm{er}}$ ordre où toutes les positions d'arguments sont des arguments d'objets.

Et encore le dernier contexte qui vaut la peine d'être analysé, vu certaines questions que l'on pourrait se poser, à savoir :

\section{7. Écrire - pisać/napisać}

a) Petits et grands ont écrit la dicté à la plume!

b) Au début du CP l'enfant écrit au crayon.

c) Ils ont écrit au bic bleu la liste des joueurs.

d) C'est un petit chef-d'œuvre sur lequel l'artiste a écrit à la main.

e) Chen Siyuan est une femme qui réussit à écrire avec ses pieds et ses mains en même temps.

Dans les exemples ci-dessus apparaissent des expressions concernant soit les objets qui servent à écrire (p. ex. : plume, crayon, bic), soit la manière d'écrire (p. ex. : à la main, avec les pieds). En ce qui concerne la manière d'écrire un contenu sur un contenant, il y a deux possibilités de l'interpréter. Si quelqu'un écrit un contenu à la main ou avec les pieds, on comprend qu'il trace des signes sur un support en employant la/les main(s) ou les pieds qui assument la fonction des objets à écrire (p. ex. en trempant les doigts dans la peinture, dans l'ancre, etc.), ou qui tiennent ces objets dans la/les main(s) ou pieds pendant l'action d'écrire. Cette deuxième possibilité de l'interprétation est métonymique : au lieu de dire p. ex. : écrire au stylo tenu à la main, on dit écrire à la main, donc on emploie la partie du corps pour l'objet qui sert à écrire.

Mais, ce qui plus est, c'est que l'on pourrait enrichir tous les exemples avec écrire analysés jusqu'à présent par des précisions avec quoi on écrit - au stylo, à la main, à la plume, etc. - p. ex. :

Les contemporains de Shakespeare ont écrit leur nom de famille de plusieurs façons différentes : au stylo, à la main, à la plume.

Il a écrit les chiffres sur un chèque au stylo, à la main, à la plume.

Il a écrit un livre sur l'imminence du conflit au stylo, à la main, à la plume.

Il est tout à fait évident que si l'on dit que Marc écrit une lettre, ce n'est pas Marc tout entier qui le fait, mais, typiquement, comme on vient de signaler cidessus, sa main qui tient un instrument d'écriture et qui représente les intentions 
de Marc. Cf. p. ex. les phrases que l'on pourrait naturellement comprendre comme un mouvement métonymique inverse par rapport à celui-ci :

\section{? Sa main/son stylo/sa plume a écrit un livre sur l'imminence du conflit.}

mais on voit tout de suite, suivant le cas, un caractère bizarre ou poétique, de toute manière métonymique, de ce type de constructions.

Alors, si l'on passe à la question fondamentale si, dans la situation d'écrire, ce avec quoi on écrit, remplit une position d'argument qui serait sémantiquement impliquée ou est plutôt un élément adjoint, on constate, vu les analyses ci-dessus, que les expressions en question ne sont que des spécifications de la manière dont quelqu'un écrit quelque chose et elles sont des éléments adjoints.

\section{Conclusion}

Après avoir analysé quelques exemples des prédicats de la liste karolakienne, il faut souligner que dans la conception de grammaire à base sémantique, la plus grande difficulté consiste à classer les prédicats selon le nombre et le type des arguments impliqués. Il est à remarquer que l'auteur de la conception propose quelques chemins à y arriver et insiste sur le manque d'une seule méthodologie qui viserait à déterminer avec précision la valence et l'ordre des prédicats (S. Karolak, 2007 : 89-95). Il est vrai qu'il accorde la primauté à la décomposition logique des prédicats en éléments sémantiquement plus simples, mais cette solution ne se montre pas toujours fiable et efficace comme outil, vu, d'un côté, la structure complexe de certains prédicats et, de l'autre, le caractère trop subjectif de l'analyse. Même s'il n'est pas facile de définir la structure conceptuelle prédicat-argument(s), on peut essayer de le faire en appliquant différents types de tests linguistiques, tels que p. ex. : paraphrase, négation (contradiction) et décomposition sémantique des sens.

Le type d'analyse proposée est possible si l'on choisit une approche déterminée parmi les trois approches générales des analyses en structures prédicat-arguments : approche du type A. Bogusławski - analyse sémantique très poussée, puisque l'on parle en fin de compte de la structure profonde ; approche du type G. Gross et sa fameuse "la suite la plus longue possible », ce qui est naturel dans le cas d'une approche distributionnelle ; et celle de S. Karolak - intermédiaire entre les deux. Nous avons adopté une position intermédiaire mixte entre l'approche d'A. Bogusławski et de S. Karolak qui est fondée et guidée principalement par une application cohérente et conséquente des paraphrases et des contradictions. 
L'important est qu'il ne faut pas oublier que la plupart des unités de la langue sont polysémiques et, ce qui en résulte, il y a souvent quelques modèles différents à proposer pour la même forme du prédicat en fonction du sens qu'il représente. Pour s'en rendre compte, il est indispensable de l'analyser toujours en contexte, puisque "You shall know a word by the company it keeps » (J. Firth, 1957 : 11). Il est nécessaire aussi de ne pas se laisser tromper par les structures superficielles de la langue où différents types d'expressions métaphoriques et métonymiques peuvent apparaître, car les apparences sont souvent trompeuses et, sous les apparences se cache la vérité à découvrir.

\section{Références citées}

Banyś, W. (2002a). Bases de données lexicales électroniques — une approche orientée objets. Partie I : Questions de modularité. Neophilologica, 15, 7-28.

Banyś, W. (2002b). Bases de données lexicales électroniques — une approche orientée objets. Partie II : Questions de description. Neophilologica, 15, 206-248.

Banyś, W. (2005). Désambiguïsation des sens des mots et représentation lexicale du monde. Neophilologica, 17, 57-76.

Banyś, W. (2019). Y a-t-il une relation entre la valence (pleine) et la synonymie ? Neophilologica, 31, 9-31.

Bogacki, K., \& Karolak, S. (1991). Fondements de la grammaire à base sémantique. Lingua e stile, 26, 309-345.

Bogacki, K., \& Karolak, S. (1992). Założenia gramatyki o podstawach semantycznych. Jezzyk a Kultura, 8, 157-187.

Bogacki, K., \& Lewicka, H. (1983). Dictionnaire sémantique et syntaxique des verbes français. Warszawa, Wydawnictwo Naukowe PWN.

Bogusławski, A. (1974). Preliminaries for semantic-syntactic description of basic predicative expressions with special reference to Polish verbs. In A. Orzechowska \& R. Laskowski (Red.), O predykacji (p. 39-57). Wrocław, Ossolineum.

Bogusławski, A. (1981). More than three or three at most? The problem of valency places and arguments of relations. Studia gramatyczne, 4, 7-14.

Bogusławski, A. (2017). W sprawie językowo-autorefleksyjnego testowania wymagań składniowych. Prace Filologiczne, LXX, 33-45.

Czekaj, A. (2013). Verbes supports ? - quelques réflexions sur la pertinence du terme. Neophilologica, 25, 7-15.

Czekaj, A. (2018). Perception et métonymie-problèmes de traduction automatique. Neophilologica, 30, 76-88.

Czekaj, A., \& Śmigielska, B. (2009). Autour de la notion de prédicat. Neophilologica, 21, $7-17$.

Danielewiczowa, M. (2010). Schematy składniowe — podstawowe kwestie metodologiczne. Poradnik Jezykowy, 3, 5-27. 
Danielewiczowa, M. (2017). Argumenty i modyfikatory — głos w dyskusji. Linguistica Copernicana, 14, 55-70.

Declés, J.-P. (1997). Systèmes d'exploration contextuelle. In C. Guimier (Éd.), Cotexte et calcul du sens (p. 215-232). Caen, Presses universitaires de Caen.

Eshkol, I., \& Le Pesant, D. (2007). Trois petites études sur les prédicats de communication verbaux et nominaux. Langue française, 153, 20-32.

Firth, J. (1957). A Synopsis of Linguistic Theory, 1930-55. Studies in Linguistic Analysis, $1-31$.

Fontanier, P. (1977 [1821-1827]). Les figures du discours. Paris, Flammarion.

Giry-Schneider, J. (1994). Les compléments nominaux des verbes de parole. Langages, $115,103-125$.

Grochowski, M. (1984). Składnia wyrażeń polipredykatywnych. In Z. Topolińska (Red.), Gramatyka współczesnego języka polskiego. Składnia (p. 213-299). Warszawa, Wydawnictwo Naukowe PWN.

Gross, G. (1993). Trois applications de la notion de verbe support. L'information grammaticale, 59, 16-22.

Gross, G. (1999a). La notion d'emploi dans le traitement automatique. La pensée et la langue, $24-35$.

Gross, G. (1999b). Élaboration d'un dictionnaire électronique. Bulletin de la Société de linguistique de Paris, XCIV(1), 113-138.

Gross, G. (2012). Manuel d'analyse linguistique. Approche sémantico-syntaxique du lexique. Villeneuve-d'Ascq, Presses universitaires du Septentrion.

Heger, K. (1969). L'analyse sémantique du signe linguistique. Langue Française, 4, $44-66$.

Hrabia, M. (2011). La grammaire à base sémantique : une conception «bâtie » et non pas « donnée ». Quelques remarques sur le changement de la compréhension de certaines notions fondamentales dans la théorie de Stanisław Karolak. Neophilologica, $23,273-289$.

Karolak, S. (1984). Składnia wyrażeń predykatywnych. In Z. Topolińska (Red.), Gramatyka współczesnego języka polskiego. Składnia (p. 11-211). Warszawa, Wydawnictwo Naukowe PWN.

Karolak, S. (1998). Sur une méthode de détermination de la valence des prédicateurs. In E. Hajičová (Ed.), Issues of Valency and Meaning. Studies in Honour of Jarmila Panevová (p. 55-61). Prague, Karolinum, Charles University Press.

Karolak, S. (2001). Założenia gramatyki o podstawach semantycznych. In S. Karolak (Red.), Od semantyki do gramatyki (p. 21-61). Warszawa, Instytut Slawistyki PAN.

Karolak, S. (2002). Podstawowe struktury składniowe języka polskiego. Warszawa, Slawistyczny Ośrodek Wydawniczy PAN.

Karolak, S. (2007). Składnia francuska o podstawach semantycznych (T. 1). Kraków, Collegium Columbinum.

Kilgariff, A. (1997). I don't believe in word senses. Computers and the Humanities, 31(2), $91-113$.

Lakoff, G., \& Johnson, M. (2003). Metaphors we live by. London, The University of Chicago press. 
Mel'čuk, I., \& Žolkovskij, A. (1970). Towards a functionning "meaning-text" model of language. Linguistics, 57, $10-47$.

Mel'čuk, I., et al. (1981). Un nouveau type de dictionnaire : le dictionnaire explicatif et combinatoire du français contemporain (six articles de dictionnaire). Cahiers de lexicographie, 38, 3-34.

Morier, H. (1998). Dictionnaire de poétique et de rhétorique (5 $5^{\mathrm{e}}$ éd.). Paris, Presses universitaires de France.

Ogden, Ch. K., \& Richards, I. A. (1923). The Meaning of Meaning. London, Routledge \& Kegan Paul.

Pozierak-Trybisz, I. (2009). Składnia francuska o podstawach semantycznych: ćwiczenia (T. 2). Kraków, Collegium Columbinum.

Przepiórkowski, A. (2017). Argumenty $i$ modyfikatory $w$ gramatyce $i$ w słowniku. Warszawa, Wydawnictwo Uniwersytetu Warszawskiego.

Śmigielska, B. (2011). La polysémie dans les dictionnaires et dans la traduction. Linguistica Silesiana, 32, 191-202.

Śmigielska, B. (2013). Le problème de la valence et de l'ordre des prédicats dans la conception des structures prédicat-arguments de Stanisław Karolak. Neophilologi$c a, 25,140-149$.

Śmigielska, B. (2019). Implication sémantique des prédicats dans la grammaire à base sémantique de Stanisław Karolak. Neophilologica, 31, 384-398.

Vetulani, G. (2000). Rzeczowniki predykatywne języka polskiego. W kierunku syntaktycznego słownika rzeczowników predykatywnych. Poznań, Wydawnictwo Naukowe UAM.

Vetulani, G. (2012). Kolokacje werbo-nominalne jako samodzielne jednostki języka. Syntaktyczny słownik kolokacji werbo-nominalnych jezzyka polskiego na potrzeby zastosowań informatycznych. Poznań, Wydawnictwo Naukowe UAM.

Vivès, R. (1993). Prédication nominale et l'analyse par verbes support. L'information grammaticale, 59, $8-15$.

Vivès, R. (1998). Les mots pour le dire : vers la construction d'une classe de prédicats. Langages, 131, 64-76.

Wierzbicka, A. (2006). Sens et grammaire universelle : théorie et constat empirique. Linx, 54, 181-207. 